\title{
Simulation of an Operating Machine in Dust Chamber
}

\author{
S. TOLLÁR ${ }^{1}$, B. BOLLó2 \\ 1University of Miskolc, Faculty of Mechanical Engineering and Informatics, Department of Fluid and Heat \\ Engineering, aramts@uni-miskolc.hu \\ 2University of Miskolc, Faculty of Mechanical Engineering and Informatics, Department of Fluid and Heat \\ Engineering, aramzb@uni-miskolc.hu
}

Abstract. Auxiliary equipment used in the automotive industry, such as generator, air conditioner, or starter, is often exposed to harsh conditions, for example splash water, frost, or serious dust load. Therefore, these auxiliary devices are intended to be prepared by the manufacturers to these conditions. The topic of this paper is a part of the analysis of the dust control of generators, which in principle is no different from the examination of other equipment. The flow around the generator and in the dust chamber was simulated by Ansys FLUENT.

\section{Introduction}

The essence of the dust test is to create an environment around the equipment to be tested, which can access the built-in auxiliary equipment in a critical area of use. The dusty environment is shaped by the chamber so that a predetermined particle size and amount of dust blows at the prescribed intervals into the chamber space, where the desired dust distribution is formed due to the formation of the chamber, where the dust then settles down. Computational fluid dynamics (CFD) also shows good potential for simulating dust flows by predicting flow distribution, stream velocity, and location of flow recirculation zones due to the effect of the dust stream on air flow [1-3].

Built into this environment, the working auxiliary equipment can be used to examine the effects of dust on the operation of the equipment. It is possible to find out where can the dust penetrate the interior of the equipment or lock off important cooling apertures or cause unwanted wear on the working surfaces.

If the equipment to be tested creates an airflow during operation, the equipment must be installed in the dust chamber in operating situation. This is also the case for generators as the generators are equipped with their own cooling fan. For the installation a mounting frame is required that meets the following requirements:

- properly positioned and fixed in the dust chamber,

- attach to the equipment to be tested (if you want to examine several types, you have the option of using different fixing points),

- allows the machine to drive (in our case: it does not hinder the belt drive),

- preferably does not affect the flow around the equipment under examination and does not cover surfaces from the settling dust. 
Planning the mounting frame therefore requires great care. We are working to make sure that our design of the built-in frame complies with our expectations before sharp industrial inspections. We must know that the result of the experiments on the dust chamber is not due to the effect of the mounting frame. This is the reason why a simulation model is needed to determine the way the design of the mounting frame. So, our goal is to create this simulation model. This article describes the phases of this model building.

\section{Dust Chamber Simulation}

The simulation test consists of the following main steps:

\subsection{Create the Geometry}

First, we need a 3D model of the dust chamber, which contains all the important details of the inside of the dust chamber, including the grid for placing the equipment to be tested. This 3D model is the space where we want to test the settling of the dust.

The full three-dimensional model of the generator was only available as an external surface model. Inner space, cooling fan blades and impeller were unavailable, so we decided to make our own threedimensional model, which includes the main geometry data of the generator, the internal flow areas, and the cooling fan impellers. This model is used for the simulation shown in Figure 1.

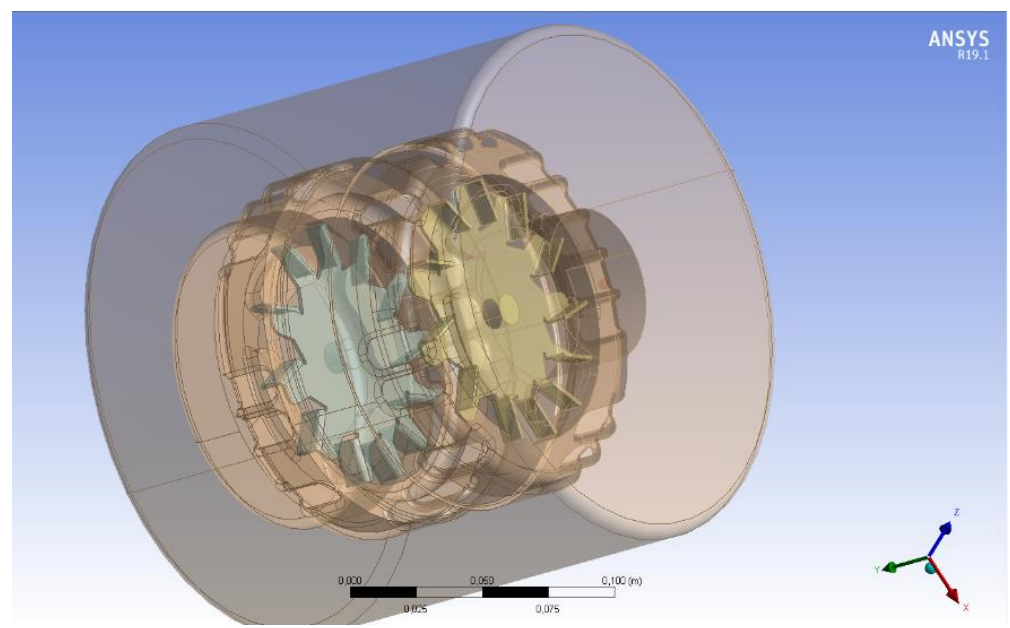

Figure 1. Generator model and near environment in ANSYS DesignModeler.

There is a need for the 3D model of the generator to be placed in the initial position in the dust chamber, ensuring its operation. This practically means that the drive shaft must be able to drive the generator and leave enough space around the walls so that the flow in the dust chamber cannot be influenced by the walls.

We must design an initial model for fixing the equipment into the dust chamber. This model shall be designed to cover as little space as possible but shall be joined on the generator's anchorages. Of course, it is also necessary to secure the mounting frame to the dust chamber grille.

If these models are available, the test space is virtually assembled. 


\subsection{CFD model}

The commercial CFD software Ansys FLUENT was used to analyse air and particle flow in the dust chamber. In the development of the numerical model, the following assumptions are made:

- the shape of dust particles is assumed to be spherical and considered as the discrete phase;

- air and other gases were used and considered as the continuum phase;

- heat and mass transfer were not considered in the simulation.

The air was considered as the continuous phase and its flow was solved based on the realizable $\mathrm{k}-\varepsilon$ model, while the dust particles were treated as the discrete phase and the trajectory of each particle was calculated by the discrete phase model (DPM).

To simulate the flow around the generator, the web is compressed in the interior of the generator and in its immediate vicinity. The total number of elements in this simulation was $\mathrm{M} 1=2.000 .000$. The mesh around the generator is shown in Figure 2.

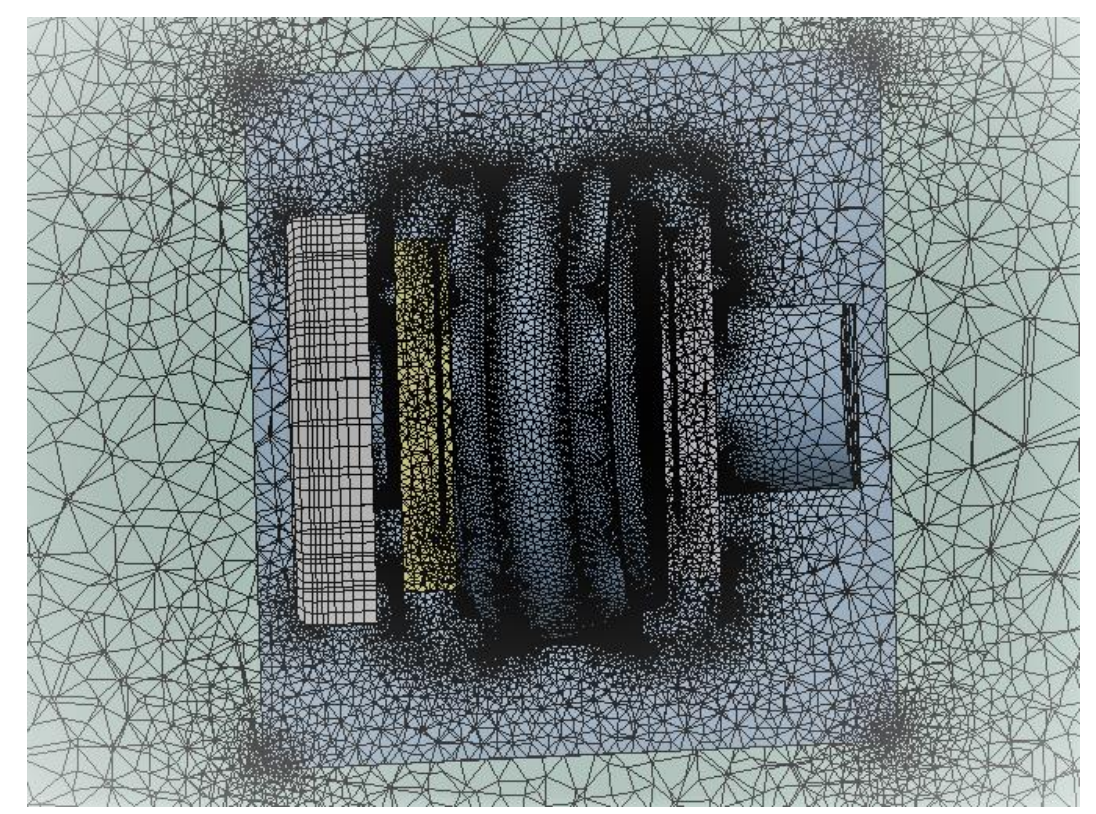

Figure 2. The mesh around the generator in ANSYS .

The simulation of the dust chamber despite the larger size, the task could be solved with fewer item counts. In this case, the mesh consisted of M2 $=700.000$ elements.

\subsection{Definition of Operating Conditions}

The simulation serves firstly to map the flows in the chamber. The model will thus be a space delimited by the chamber walls, which must define the cross-section for the air supply, the outlet section, the airflow volume and the temperature of the air.

To run the simulation, we need data. We need to define the working point of the fan of the dust control. This was determined by the measurement of the colleagues in the absence of technical data. The fan working point can be described with the following values: 


$$
\begin{gathered}
Q=340 \mathrm{~m}^{3} / \mathrm{h}, \\
\Delta p_{t}=920 \mathrm{~Pa}, \\
\Delta p_{s t}=850 \mathrm{~Pa}
\end{gathered}
$$

The temperature of the air is determined by the test description: $80^{\circ} \mathrm{C}$, so $353.15 \mathrm{~K}$.

For accurate simulation however, it is essential to consider the flows from the generator's operation. The generator has its own cooling fan, which rotating at the generator speed. Consequently, in different operating states means different degrees of flow around the generator. We have been selected two characteristic speeds for the tests: RPM 3000 and RPM 6000.

The simulation of the flow generated by the generator's own fans is a more complex task than the simulation of the dust chamber, so we needed to search a suitable solution for taking into account the flows generated by the generator fans.

Our chosen solution was to measure the flows formed by the rotating generator around the characteristic rotation of the generator by CTA flow measurement. The CTA anemometer works based on convective heat transfer from a heated sensor to the surrounding fluid, the heat transfer being primarily related to the fluid velocity. By using very fine wire sensors placed in the fluid and electronics with servo-loop technique, it is possible to measure velocity fluctuations of fine scales and of high frequencies. The advantages of the CTA over other flow measuring principles are ease-of-use, the output is an analogue voltage, which means that no information is lost, and very high temporal resolution, which makes the CTA ideal for measuring spectra.

Based on the measurement results we decided to generate the velocity field with the simulator around the generator. So, we will use the measurement data to validate the simulation. The simulation space is complete. Input data are also available. So, we can start to make the simulation model.

\section{Results}

The volume of the dust chamber is about 1.300 times the volume of the generator and about 5.000 times the volume of the fan environment, so that we need to see that the fineness of the network simulating the operation of the generator fan and the physical size of the dust chamber and the simulation of the dust blower require different mesh size, which is not qualitatively permissible in the simulation. With the cell sizes used in the generator's flow space, the total encoder size would represent a computational capacity that exceeds our potential.

Based on our previous investigations, it can be stated that the generator fans operate only in the very small environment of the fan, so the dust chamber tests are divided into two parts.

\subsection{Simulation of Flow of the Generator Fan}

One of the tests covers the vicinity of the generator, including a mounting bracket. This simulation can be performed with a reasonable number of elements and can be determined whether the mounting frame influences the flows around the generator. To perform the simulation, pre-simulations were 
performed with one side fan of the generator. The space around the generator has been narrowed to a size where we have experienced appreciable flows at the measurements.

\subsubsection{Pre-simulation with one side of Generator}

This simulation was assumed by transient flow conditions to be very small, with $3.33 \cdot 10^{-4} \mathrm{~s}$ time step and a large iteration number to reach the convergent result. When the number of iterations fell below 15 , the time step was taken in several steps for $0.1 \mathrm{~s} .43 \mathrm{~s}$ real time simulation was completed. It was evaluated, and we found that the flow reaches the permanent status very fast, in 0.6 seconds real time and then practically did not change. The resulting flow can thus be virtually time-constant, meaning that the simulation of the full generator with both running fans and the built-in frame can now be used to start the calculations.

The quantified speeds were compared in discrete points with the CTA measurement results. We have found that the simulation results and the measured values correspond to each other. Thus, this part of a simulation can be considered validated. For some of the typical points the measured air velocities were compared to the CFD results at RPM 3.000 in Table 1.

\begin{tabular}{|c|c|c|c|c|c|}
\hline \multirow{2}{*}{$\begin{array}{c}\text { RPM } \\
3000\end{array}$} & \multirow{2}{*}{ Position } & $U_{\text {Mean }}$ & $V_{\text {Mean }}$ & $C_{\text {Mean }}$ & $C_{\text {Calc. }}$ \\
\cline { 2 - 6 } & & \multicolumn{4}{|c|}{$[\mathrm{m} / \mathrm{s}]$} \\
\hline \multirow{3}{*}{ Inlet } & $\mathrm{P}_{1}$ & 4.154 & 1.058 & $\mathbf{4 . 2 8 7}$ & $\mathbf{4 . 2 5 2}$ \\
\cline { 2 - 6 } & $\mathrm{P}_{2}$ & 3.378 & -1.212 & $\mathbf{3 . 5 8 9}$ & $\mathbf{3 . 6 1 5}$ \\
\hline \multirow{3}{*}{ Outlet } & $\mathrm{P}_{3}$ & 2.954 & -0.536 & $\mathbf{3 . 0 0 2}$ & $\mathbf{3 . 0 8 7}$ \\
\cline { 2 - 6 } & $\mathrm{P}_{4}$ & 3.631 & -0.205 & $\mathbf{3 . 6 3 7}$ & $\mathbf{3 . 5 8 1}$ \\
\cline { 2 - 6 } & $\mathrm{P}_{5}$ & 8.767 & 0.005 & $\mathbf{8 . 7 6 7}$ & $\mathbf{8 . 7 2 0}$ \\
\hline
\end{tabular}

Table 1. Measured and calculated velocity values in some characteristic points.

These results relate only to the magnitude of the velocity, because the CTA measurements could not determine the velocity direction. Therefore, it was necessary to simulate the flows around the generator, despite our preliminary assumption. In the result of the simulation, speed directions - speed vectors - also appear that are not perpendicular to the exit surfaces. So, it was right that we decided not to put the velocity values from the measurement results on the outgoing surfaces in the direction perpendicular to the surface. This would have resulted in incorrect speed distribution.

The velocity distributing around the generator which generated by the generator's own cooling fan are shown in Figures 3., 4., and 5. 
International Journal of Engineering and Management Sciences (IJEMS) Vol. 4. (2019). No. 1 DOI: 10.21791/IJEMS.2019.1.34.

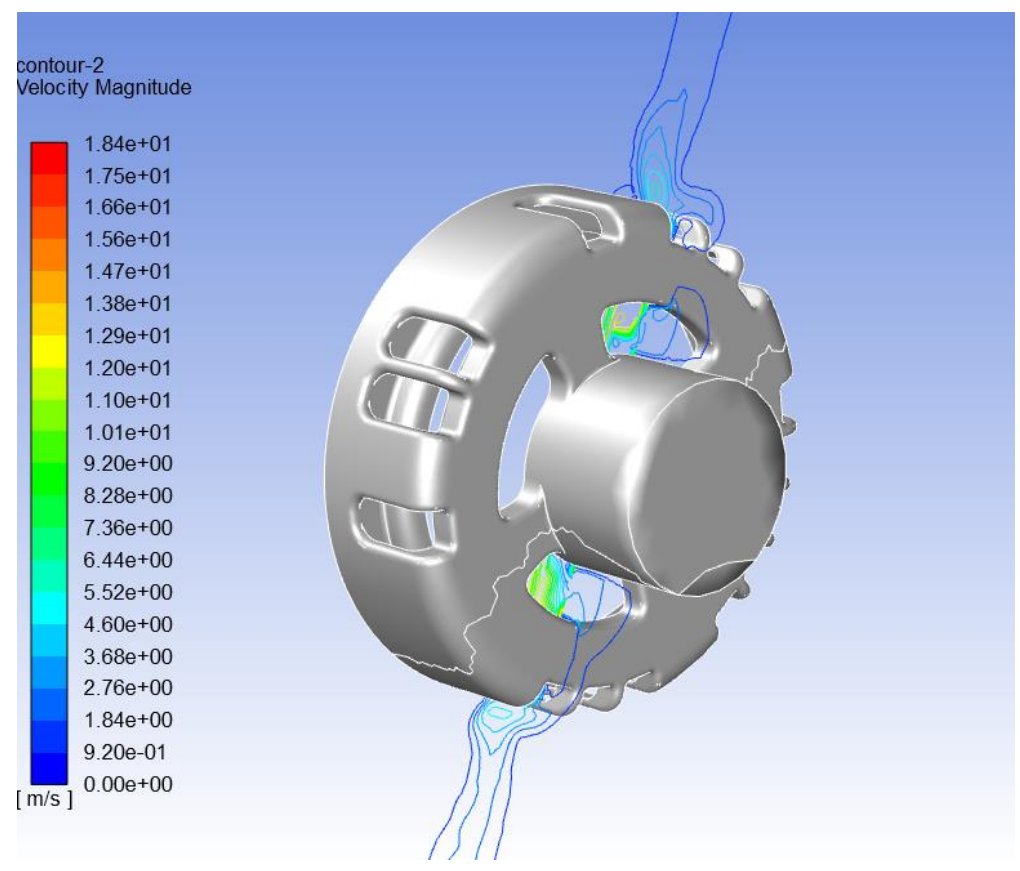

Figure 3. Evolution of velocity around the front of the generator in a vertical plane.

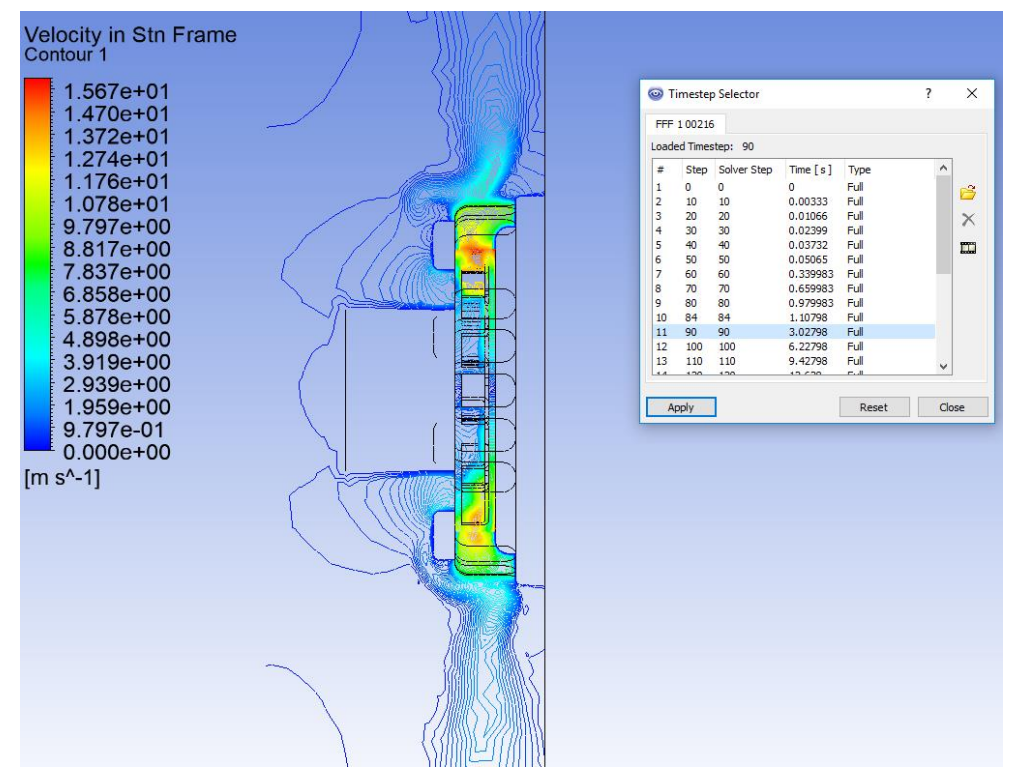

Figure 4. The constant velocity state around the front of the generator in a vertical plane after 3 seconds of real time operation. 


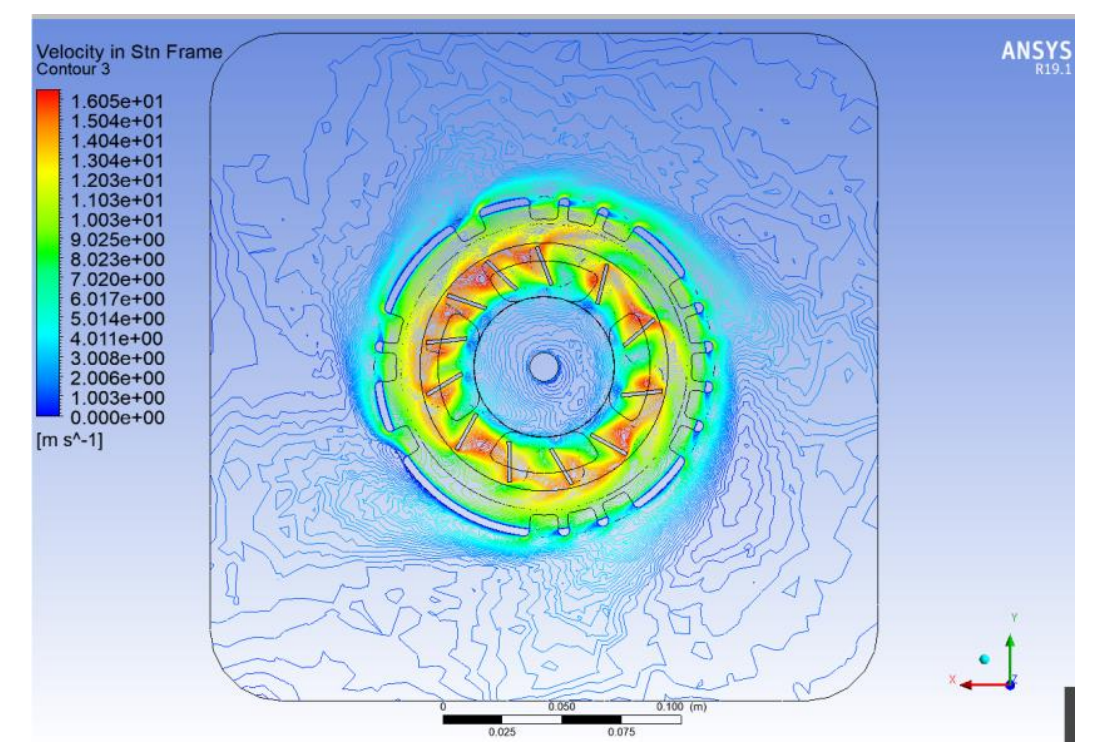

Figure 5. Constant velocity state in the plane perpendicular to the axis of the generator at the front of the generator after 3 seconds of real-time operation.

\subsubsection{Simulating the environment of the generator with both fans}

In this case, the full generator model was placed at the mounting position of the mounting bracket. The mounting frame designed by the colleagues was slightly simpler for the simulation, because we did not want to display the holes, surfaces and fixing elements that are irrelevant to the simulation. This would require additional cell numbers and more complicated meshing, but our simulation results would have no effect. The simulation model is shown in Figure 6.

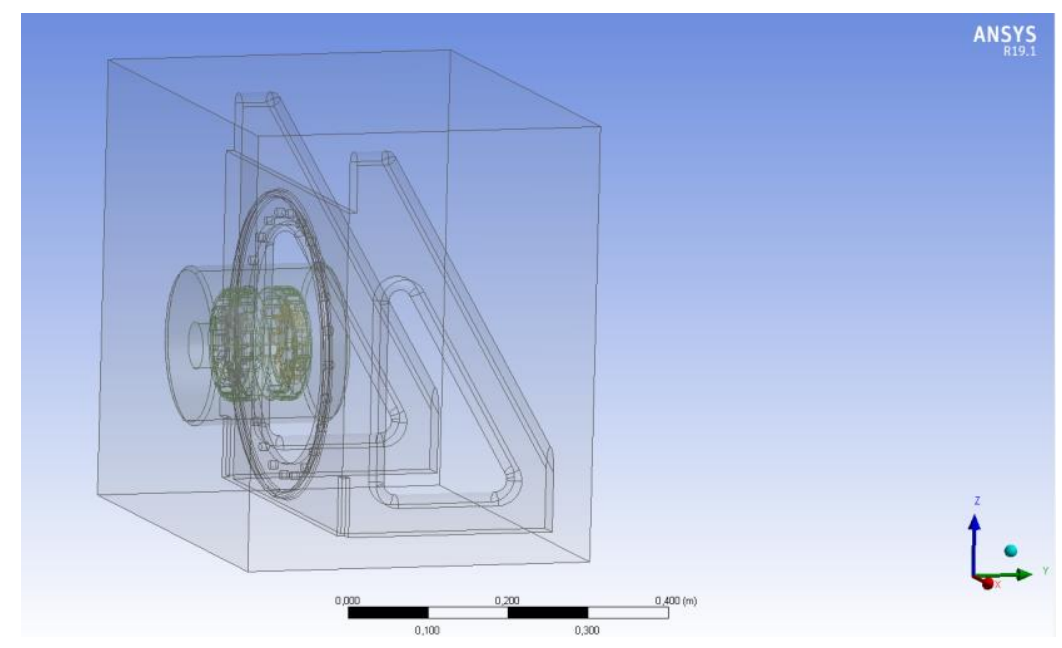

Figure 6. Generator and enclosure frame for fan simulation.

During the simulation, using the experiences of the pre-runs, both fans are used to look at the impact of the locking frame. 


\subsection{Simulation of the Dust Distribution in the Dust Chamber}

For the other simulation, the dust distribution in the whole dust chamber is tested without the generator fans being operated, so the generator is only a surface model in the simulation. Thus, meshing does not require extreme fineness in the environment of the generator and thus we can examine whether the mounting frame changes the dust distribution in the dust chamber near the generator. This test involves examining two cases. Determination of the dust distribution of the dust chamber without the mounting frame or with the mounting frame. However, in both cases, the generator is positioned in the dust chamber.

We also started simulation with a pre-simulation. We did not put a model in the dust chamber, while we just wanted to determine the settings for dust blowing and dust distribution simulation. That is why we can achieve a good result in a much shorter time with a much rougher meshing.

The blown dust for simulation of dust distribution is fed into the simulation according to ISO 12103-1 [4] fraction distribution. In this case five different fractions are distinguished. Each fraction being considered by the average particle size and the fraction weight of the total weight per fraction. By the fractional mass flow per second calculated, and the total $2 \mathrm{~kg}$ loading within 1 second being inserted into the chamber, because in the testing of the dust chamber it has been found that the blower fan blows the total amount of dust in very short time. The standard fractions or the values used for the simulation are shown in Table 2.

\begin{tabular}{|c|c|c|c|}
\hline \multicolumn{2}{|c|}{ ISO 12103-A2 for fine test dust } & \multicolumn{2}{|c|}{ Modelled dust } \\
\hline $\begin{array}{c}\text { Size } \\
{[\mu \mathrm{m}]}\end{array}$ & $\begin{array}{c}\text { Mass fraction } \\
{[\%]}\end{array}$ & $\begin{array}{c}\text { Size } \\
{[\mu \mathrm{m}]}\end{array}$ & $\begin{array}{c}\text { Flow rate } \\
{[\mathrm{kg} / \mathrm{s}]}\end{array}$ \\
\hline 0 to 5 & $39 \pm 2$ & 2.5 & 0.78 \\
\hline 5 to 10 & $18 \pm 3$ & 7.5 & 0.36 \\
\hline 10 to 20 & $16 \pm 3$ & 15 & 0.32 \\
\hline 20 to 40 & $18 \pm 3$ & 30 & 0.36 \\
\hline 40 to 80 & $9 \pm 3$ & 60 & 0.18 \\
\hline
\end{tabular}

The blow velocity by the inlet cross section was determined at $3 \mathrm{~m} / \mathrm{s}$ based on the colleagues' measurements. The simulation was running as a transient process with time step of $0.01 \mathrm{~s}$. We have found that if we show 50-50 particle tracks per $1 \mathrm{~s}$, the trails are filled up into the interior of the dust chamber. This is consistent with the visual experience that the dust in the dust chamber completely distributed in a short time. For illustration purposes, only 2-2 particle traces are shown after $0.5 \mathrm{~s}$ in the Figure 7.

After the pre-simulation, the generator and the mounting frame were placed in the dust chamber. This layout is shown in Figure 8.

At the meshing this configuration we have received a much larger computing capacity requirement even without running the fans. The chamber grid which holding the mounting frame was not shown in this simulation. Because the grid has an open surface of the total cross-section compared to nearly 
97\%. However, it would have had a considerable impact on meshing and would therefore have raised the need for computing capacity unjustified. Later we plan to consider grid as a porous surface.

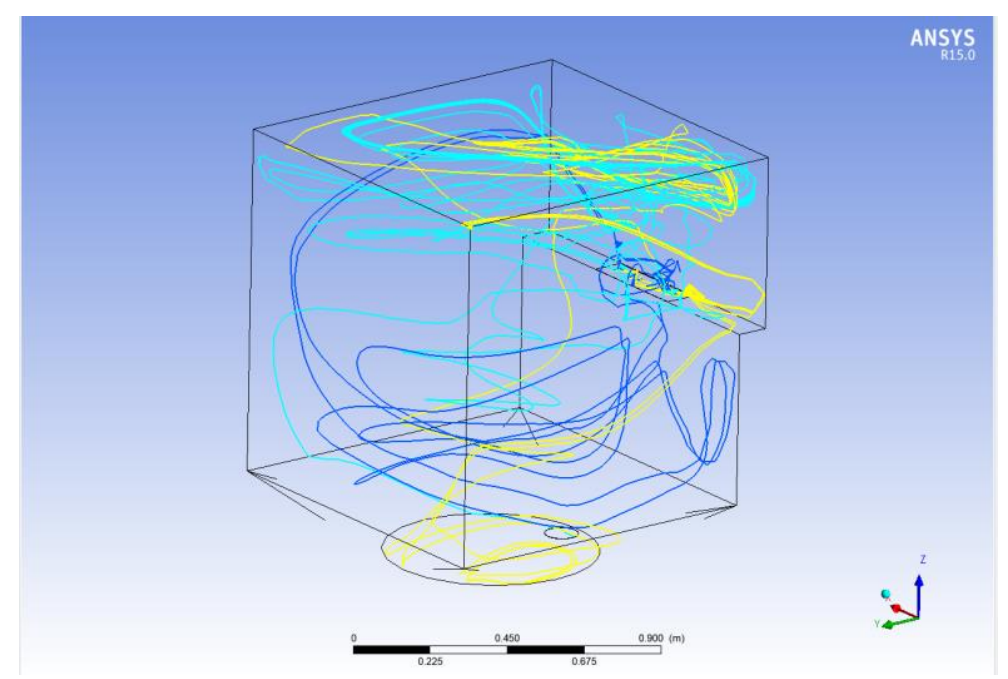

Figure 7. The path of different factions is fed for 0.5 seconds after starting the blow.

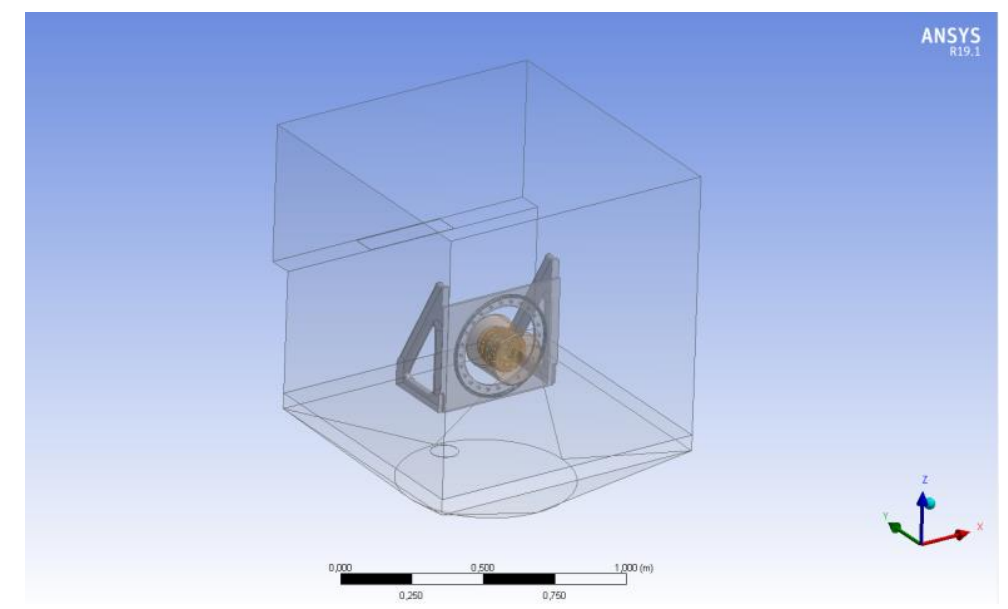

Figure 8. Model of dust chamber with mounting frame and generator.

\section{Conclusions}

The flow generated in the environment of the generator was simulated and the data were compared with the CTA measurement data. We have found that our simulation model is suitable for simulating flows around the generator, as well as for examining the effect of the mounting frame. Based on our present study, it can be said that the mounting frame does not significantly alter the flow around the generator.

We also simulated the distribution of the blown dust in the dust chamber. Comparing our simulation results with those in the working dust chamber, we found that our simulation is a good approximation of reality, so it is suitable for simulation of dust distribution. We have found that the mounting frame does not significantly affect the distribution of dust in the dust chamber. With our previous finding, we 
found that the mounting frame is suitable for incorporating the generator and conducting the tests in the real dust chamber.

All in all, we have solved a complex task, which can be the starting point for subsequent similar studies. We have come up with a step forward: the creation of a simulation model is a goal in which simultaneously the dust distribution and flows from the operation of the fans can be displayed. The difficulty is represented by considerably different geometric dimensions. So further investigations are needed in this field.

This research was supported by the European Union and the Hungarian State, co-financed by the European Regional Development Fund in the framework of the GINOP-2.3.4-15-2016-00004 project, aimed to promote the cooperation between the higher education and the industry.

\section{References}

[1] W. Devarrewaere - U. Heimbach - D. Foqué - B. Nicolai - D. Nuyttens - P. Verboven (2016) Wind tunnel and CFD study of dust dispersion from pesticide-treated maize seed. Computers and Electronics in Agriculture. 128 pp. 27-33.

[2] X.L. Chen - C.A. Wheeler - T.J. Donohue - R. McLean - A.W. Roberts (2012) Evaluation of dust emissions from conveyor transfer chutes using experimental and CFD simulation. International Journal of Mineral Processing. 110 pp. 101-108.

[3] F. Geng - G. Luo - Y. Wang - Z. Peng - S. Hu - T. Zhang - H. Chai (2018) Dust dispersion in a coal roadway driven by ahybrid ventilation system: A numerical study. Process Safety and Environmental Protection. 113 pp. 388-400.

[4] ISO 12103-1: Road vehicles - Test contaminants for filter evaluation - Part 1: Arizona test dust (2016) 